ISSN 1991- 8690

Website: http://jsci.utq.edu.iq

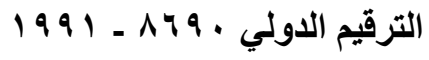

Email: utjsci@utq.edu.iq تأثير مطروحات معمل النسيج في تراكيز بعض العناصر النزرة في مياه نهر الفرات عند مركز مدينة الناصرية - جنوب العرلق

$$
\text { حسنين علي الكناني }
$$

$$
\begin{aligned}
& \text { تضمنت الدراسة بيان أثر المطروحات الصناعية الناتجة من معمل المنسوجات الصوفية في تراكيز ستة من العناصر النزرة (الكادميوم، النحاس، }
\end{aligned}
$$

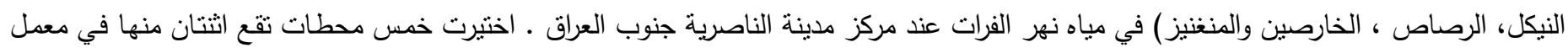

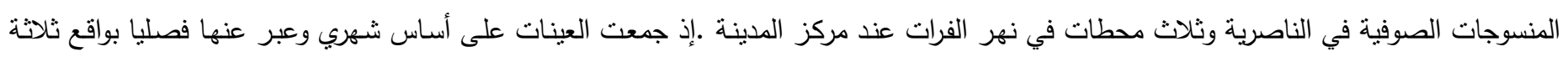

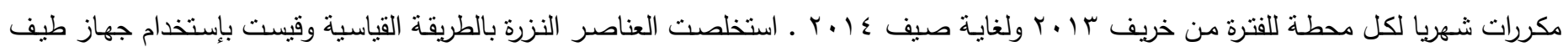

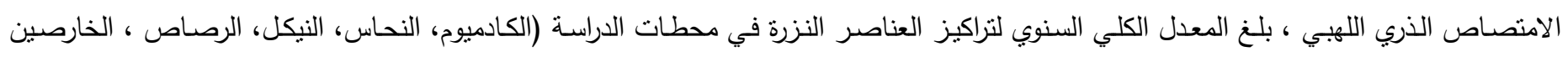

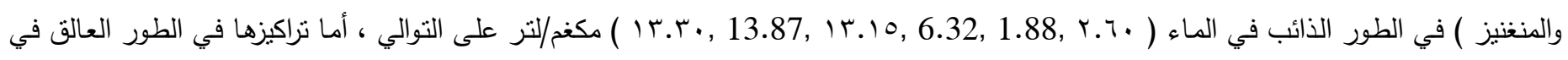

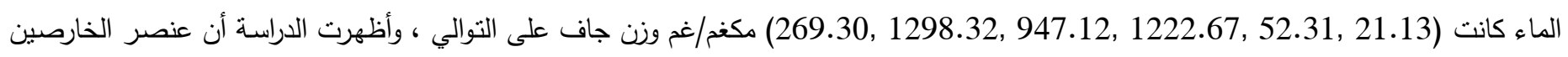

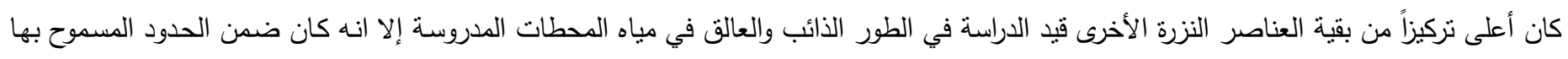

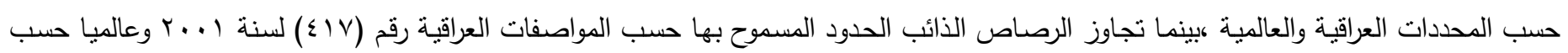

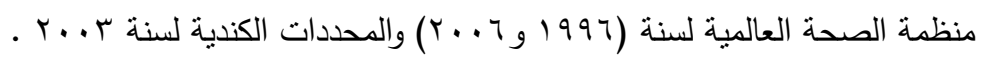

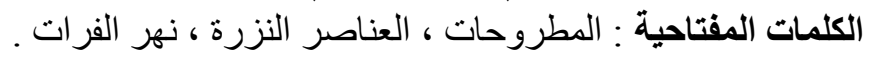

\title{
Effect of Textile Factory effluents upon concentrations of Some Trace Elements in Water of the Euphrates River at the center of Al-Nasiriyah city Southern Iraq
}

\author{
Bassim Y.AL-Khafaji
}

Hasanain A.AL-Kinany

\section{Abstract:}

Dept. of Biology - Coll. of Science - Univ. of Thi-Qar

The present study deal with the effect of the effluents from of Textile Factory, in concentrations of six trace elements ( Cadmium, copper, Nickel, Lead, Zinc, and Manganese) in water of Euphrates river at the center of AlNasiriyah south of Iraq. Five stations have been chosen, two of them where are located at the Textile Factory in ALNasiriyah, and three of these are located at the Euphrates river at the city center. That that samples were collected monthly as triplicates from each station and expressed seasonally, for the period from Autumn 2013 until Summer of 2014 . Trace elements are extracted by a standard method and measured by using flamed atomic absorption spectrophotometer. The mean of total concentration were ( Cadmium, Copper, Nickel, Lead, Zinc, and Magnesium) in the dissolved phase of water were $(2.60,1.88,6.32,13.15,13.87,13.30) \mu \mathrm{g} / \mathrm{L}$, respectively. But its concentration in the suspended phase in water were $(21.13,52.31,1222.67,947.12,1298.32,269.30) \mu \mathrm{g} / \mathrm{gm}$ dry weight, respectively. This study shows up that Zinc elements was in more than concentration of there trace elements under study in the dissolved and suspended phases of the studied stations. But, it is found under the permitted limits according to the international and Iraqi limitations, while the lead in dissolved phase more than the permitted limitation according to the 
Iraqi qualifications No(417) in 2001 and internationally according the World Health Organization in (1996 and 2006) and the Canadian Limitations in 2003.

Key words: Effluents, Trace Elements , Euphrates River

أثنار موسى و علي ( 1985) إلى أن مخلفات معمل آخر للغزل والنسيج في الكاظمية في بغداد ايضاً كانت تراكيز عنصر الكران الكروم منخفضة في اغلب المحطات باستثناء تراكيزه في الفضلات المصرفة فئنا

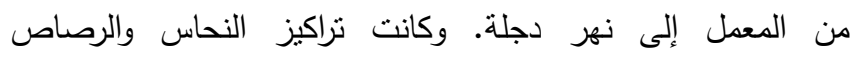
والكادميوم مرتفعة في اغلب المحطات وتتعدى الحدود المسموح بها

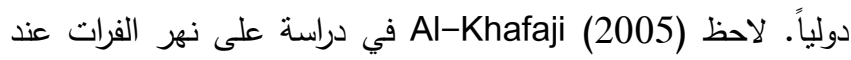
مدينة الناصرية جنوب العراق ارتفاع في تراكيز بعض العض العناصر

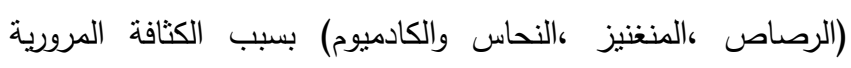

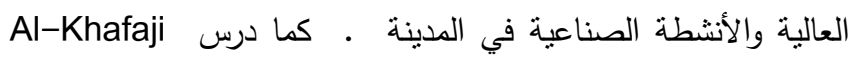

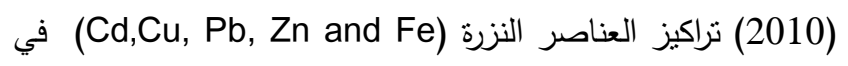
نهر الفرات قرب مركز مدينة الناصرية إذ بلغت معدلاتها في الماء

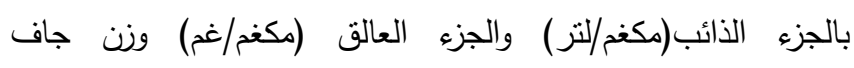
كالأتي (0.15 , 16.13)، (0.59 , 24.48)، , 0.20 (909.4) (726،( 0.20 , 49.95)، ( 2.5 , 35.62) على التوالي • وبيّن

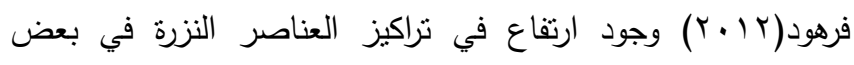

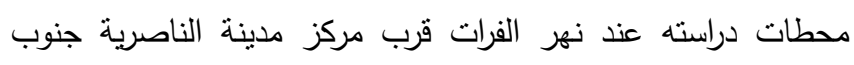
العراق والتي تكون واقعة تحت نأثير مطروحات الطاقة الكهربائية

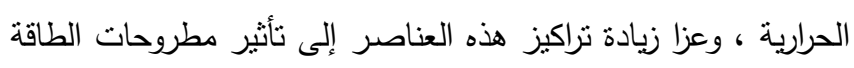

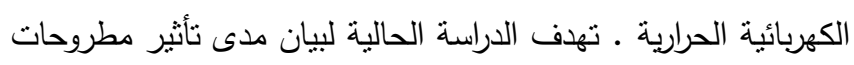

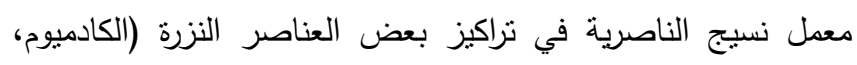

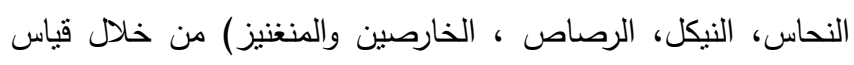
تراكيزها في مياه نهر الفرات عند مركز مدينة الناصرية.

\section{وصف منطقة الدراسة:}

يعـد معدل المنسـوجات الصـوفية في الناصـرية احــ المعامـل

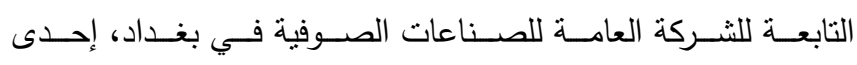
تشكيلات وزارة الصناعة والمعادن ، يقع في منطقة صناعية مجاورة

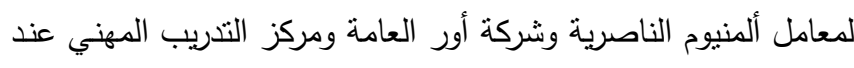
جنوب محافظة ذي قار قرب مركز مدينة الناصرية وبإحداثيات (شمال

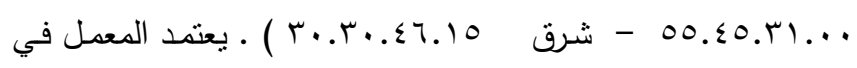

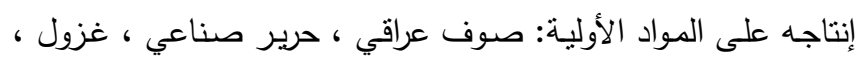

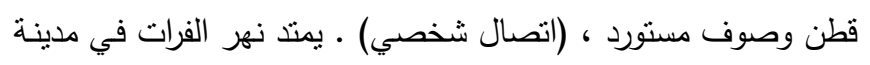

المقدمة :

يعد التلوث بالمخلفات الصناعية من اخطر مصادر تلوث المياه

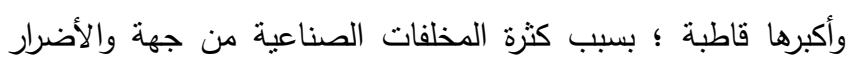
الكبيرة القاتلة للكائنات الحية من جهة أخرى ، ومن أخطرها التلوث

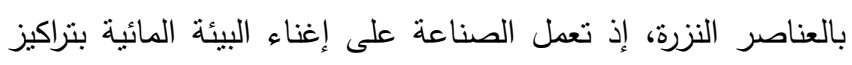

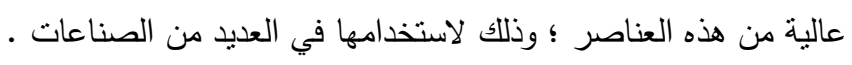
تأتي خطورة هذه العناصر من عدم قدرة النهر على التخلص منها ذانياً

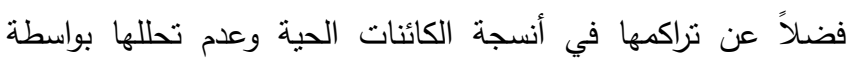
الأحياء المجهرية ، ويمكن أن يتضاعف نركيزها بوساطة السلسلة

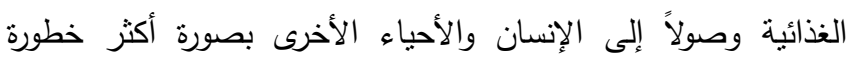

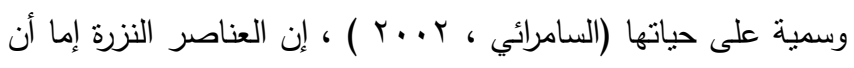

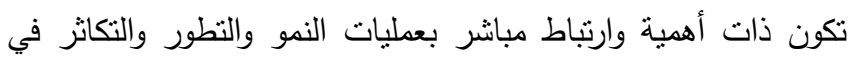
الكائنات الحية مثل المنغنيز، الكوبالت، النحاس،الحديد و واتئر الخارصين

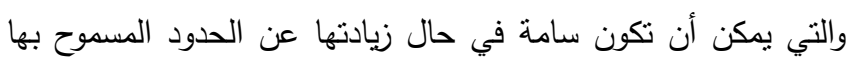

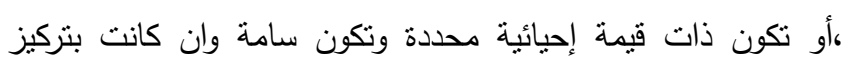

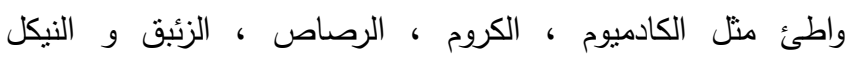
( MDE , 2003 ; Tokalioglu et. al., 2000)

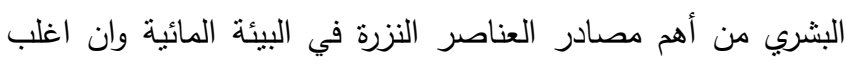

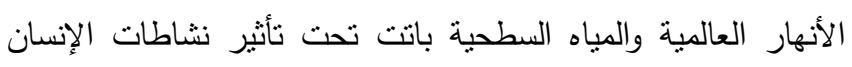

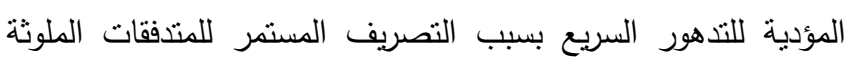
ويمنل النشاط البشري جميع المصادر الناشئة عن فعاليات الإنسان

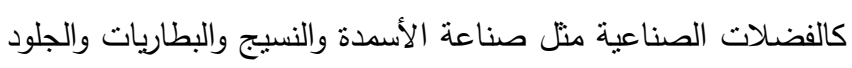
والأصباغ ونواتج معامل تكرير النفط ، وتؤدي الفضلات المنزلية دوراً

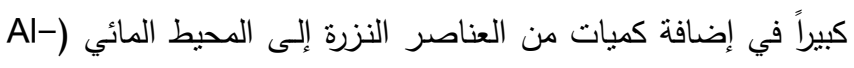

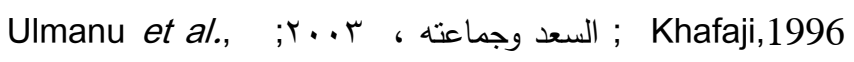
2003 ؛ Percy , 2004 ) · وهناللك عدة دراسات محلية بحثت في مدى تأثير الأنشطة الصناعية وما تطرحه من العناصر النزرة في البيئة

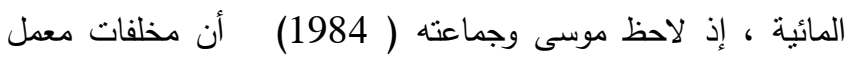

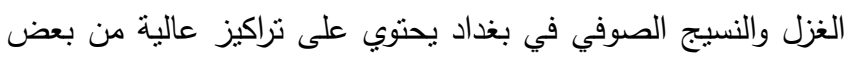

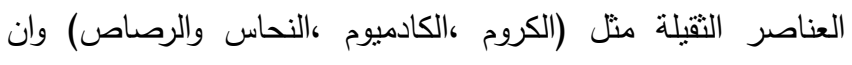

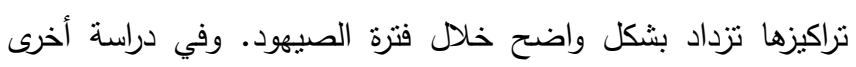




\section{استخلاص العناصر النزةة

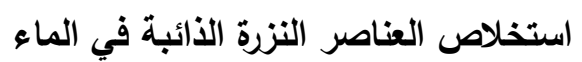

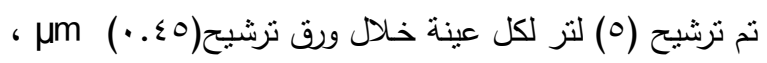

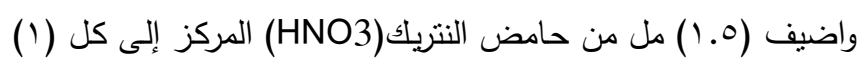

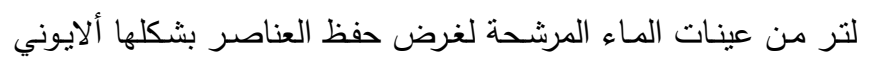

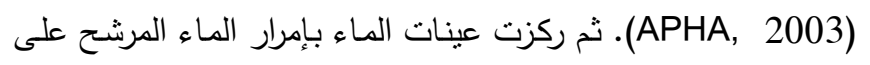

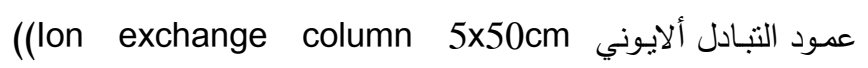
الحاوي على راتنج نوع (Chelex-100) بالثكل الصوديومي وبسرعة لانزيد عن (0) مل/دقيقة (Riley \& Taylor, 1968) • استخدام

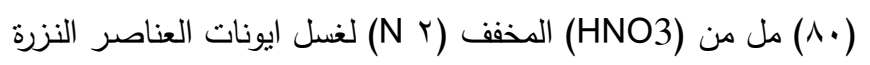

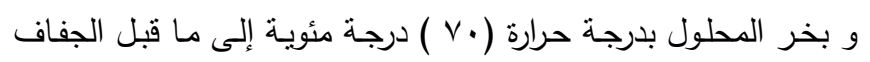

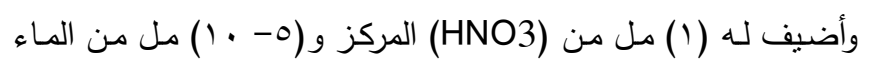

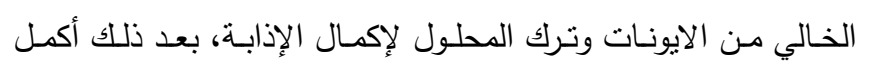

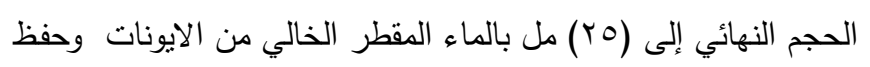

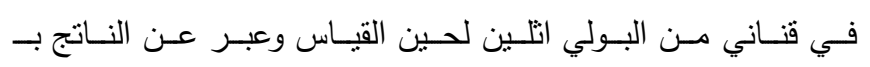
مايكروغرام/لتز (APHA, 2003).

\section{استخلاص العناصر النزدة العالقة في الماء}

جفت أوراق الترشيح المستعملة لترشيح عينات الماء في بدرجة

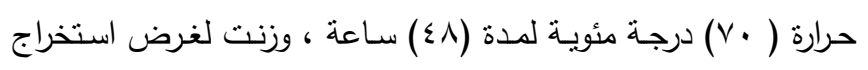

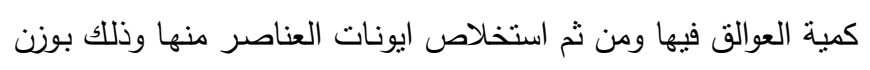

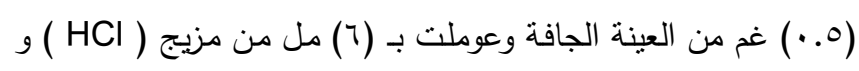

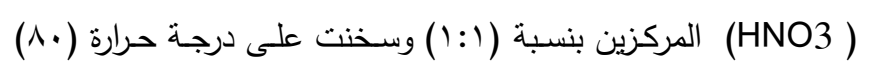

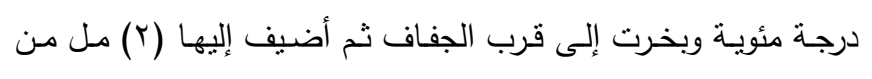

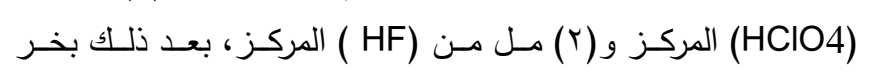

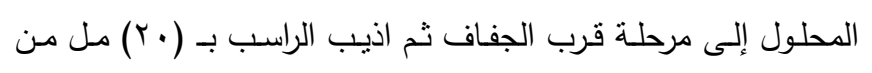
وأضيف حجم معين من الماء المقطر خالي من (0.5 N) ( HCl)

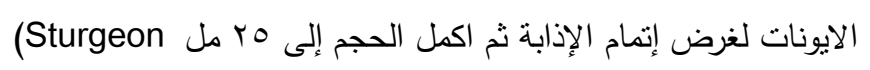
وحفظ في قناني من البولي انلين لحين القياس وعبر الدير عن الناتج بـ (مايكروغرام/غرام وزن جاف) .

\section{قياس أيونات العناصر النزرة:}

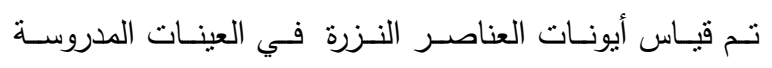

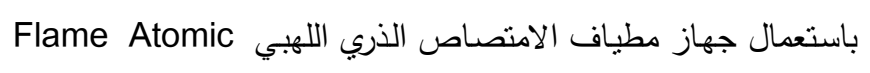
Absorption Spectrophotometer

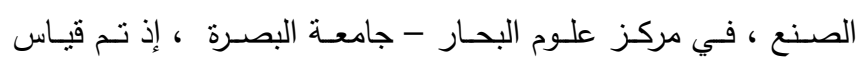

الناصرية بطول حوالي( or.o) كم من دخولـه عند ناحية البطحاء

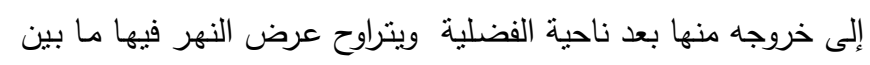

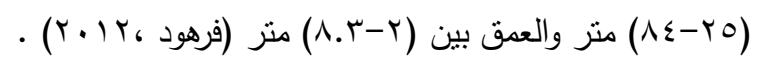

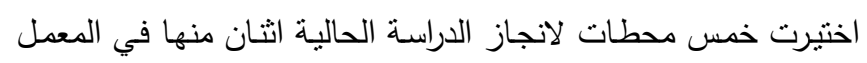

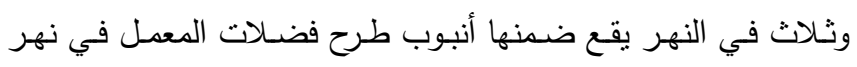

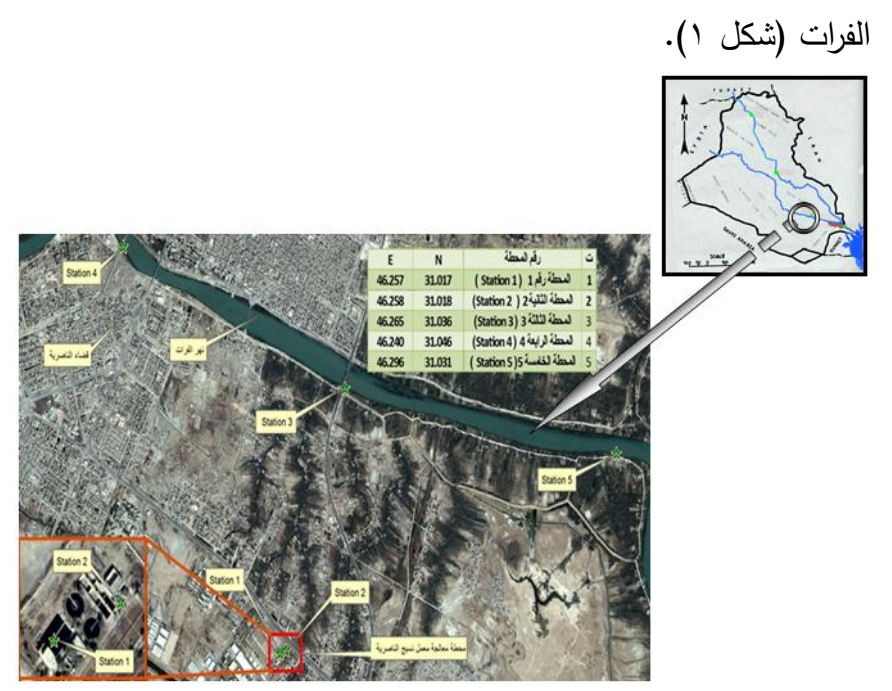

شكل(1) خريطة العراق توضح نهر الفرات مع صورة جوية للنهر عند مدينة

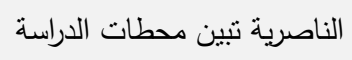

\section{المواد وطرائق العمل :}

جمع العينات

جمعت عينات المساء بأستخدام قناني بلاستيكية (بولي أنلين)

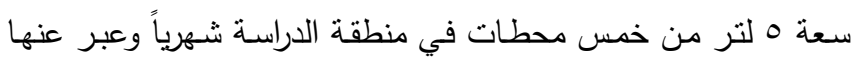

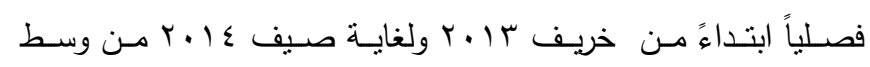

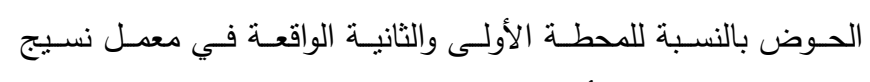

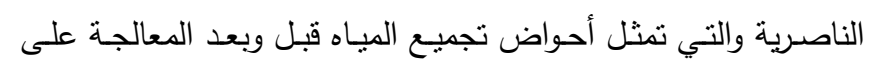

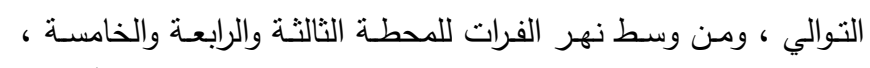

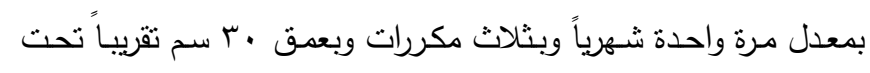

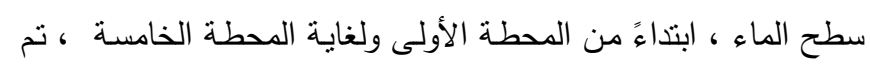

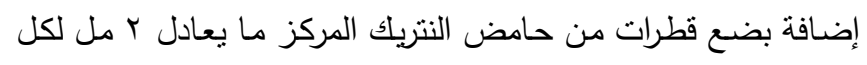

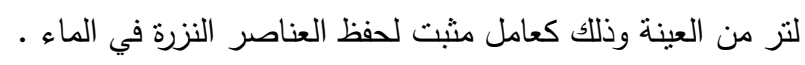


وتبين من التحليل الإحصائي وجود فروق معنوية منباينة بين المحطات

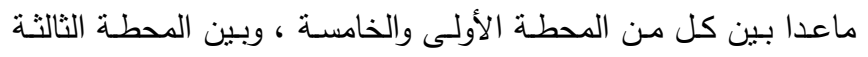

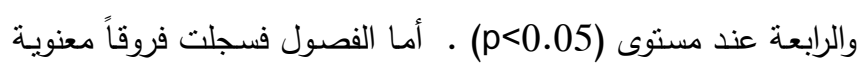
فيما بينها عند

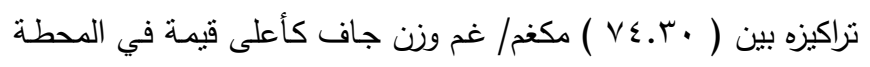

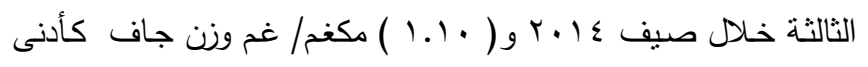

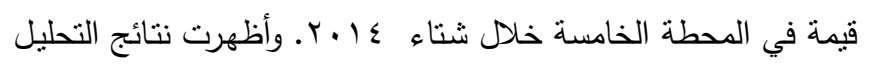

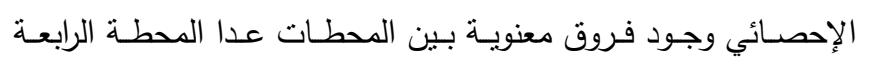
والخامسة لم تسجل بينهما فرقاً معنوياً عند مستوى (P<0.05) ، أمسا الفصول فقد سجلت

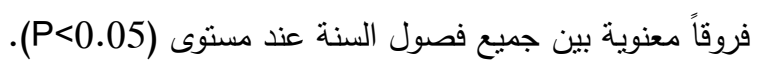

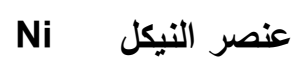

تراوحت قيم النيكل مسن أعلى قيمسة في الطسور الذائب (

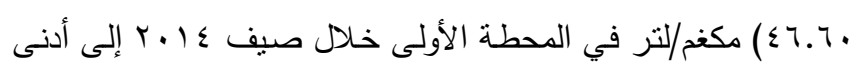

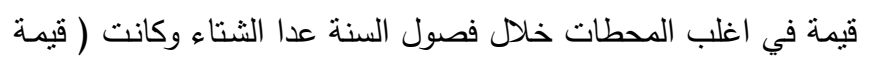

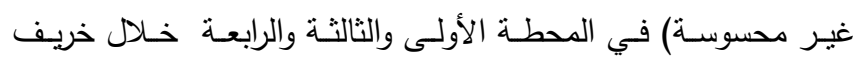

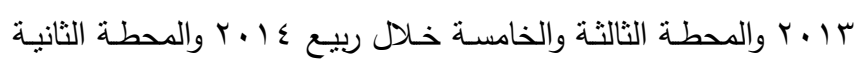

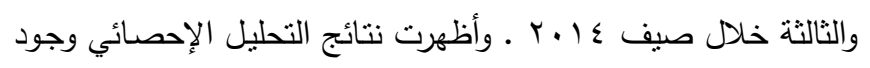

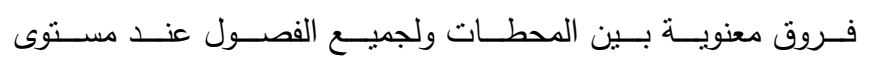

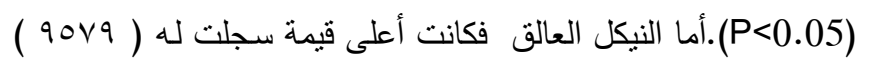

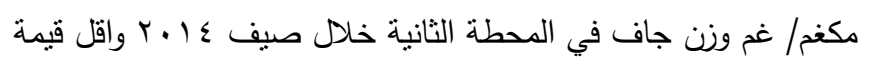

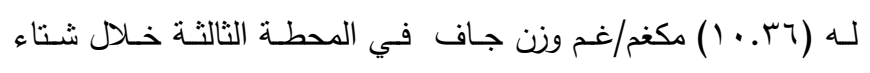

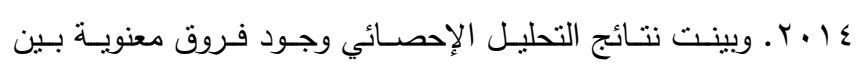

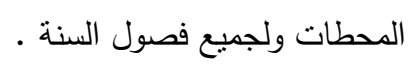

عi عن الرصاص

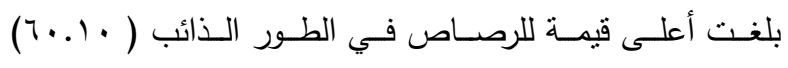

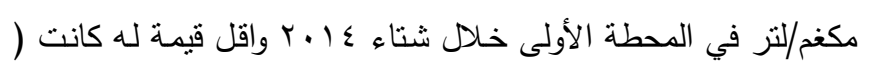

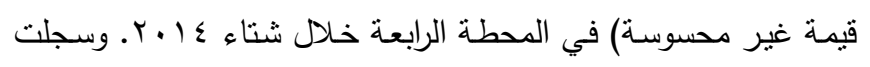

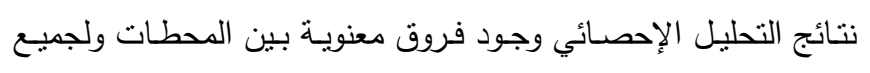

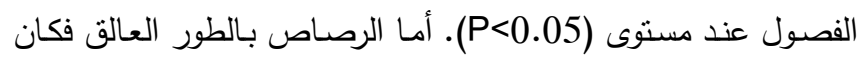

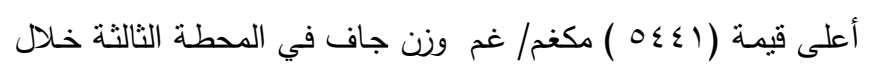

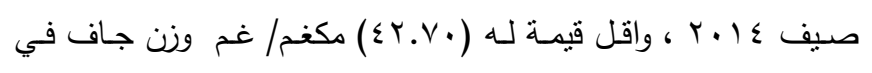

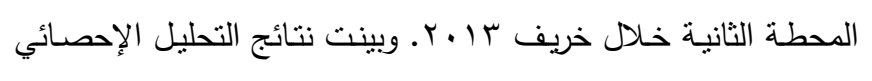

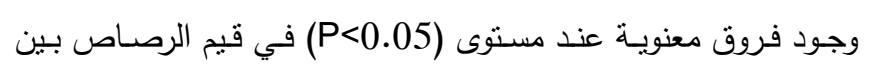

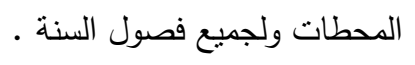

عناصر (الكادميوم و النيكل والرصاص والخارصين والمنغنيز والنحاس)

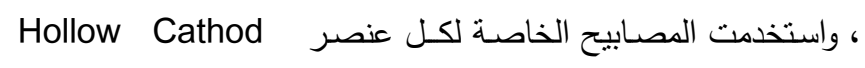

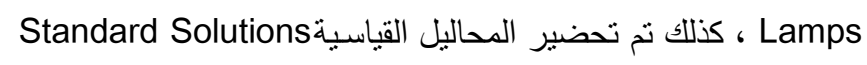

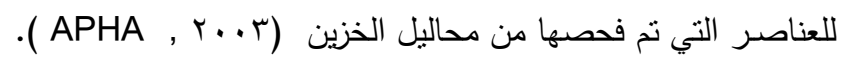
حسبت تراكيز العناصر النزرة من منحنى المعايرة وفق المعادلة الآتية و الموضحة من قبل ( Al-Khafaji,1996). معادلة حساب العناصر النزرة الذائبة في الماء : AxB $A \times B$ Econ $=\longleftarrow \quad \ldots$ C

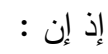
= E $=$ Con = تركيز العنصر المستخرج من منحني المعايرة (ملغم/لتز)

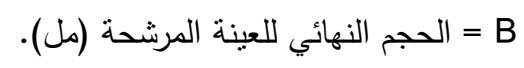
= الحجم الابتدائي للعينة المرشحة (مل).

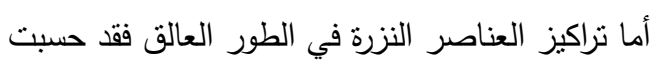

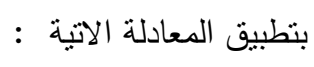
$A \times B$

$$
E \text { con }=\frac{}{D}
$$

: : إذ ECon. = تركيز العنصر المستخرج من منحني المعايرة (ملغم/لتز) ) A

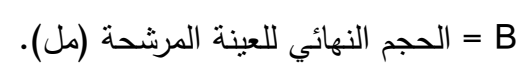
= D

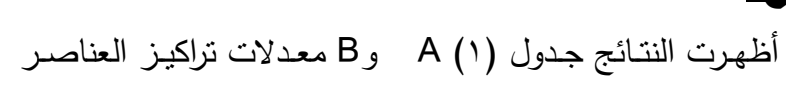
النزرة في الماء بطوريه الذائب والعالق وتم تحليلها احصائيا باستخدام برنامج 10 SPSS وكما يأني:

\section{Cd عنصر الكادميوم} أن أعلى قيمة للكادميوم الذائب ( • • V. ) مكغم/لتر في المحطة

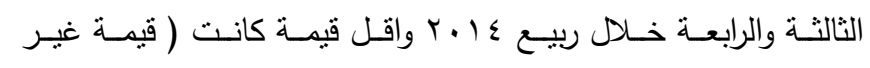

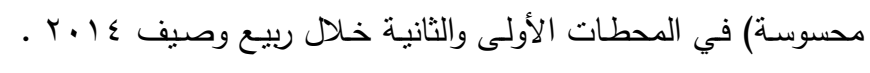




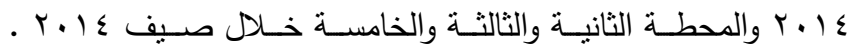

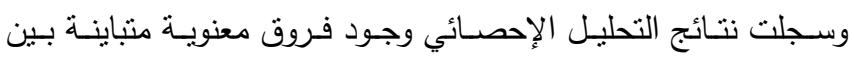
المحطات إذ لم تسجل فروقاً معنويـة بين المحطة الأولى والخامسـة ،

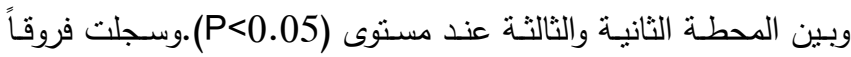
معنوية بين جميع فصول السنة عند المستوى نفسه. أما المنغنيز بالطور العالق فكان أعلى قيمة لـ(917 ) مكغم/ غم وزن جاف في الدحطة

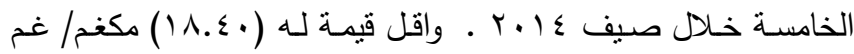
وزن جاف في المحطة الثانية خلال شتاء ع ا ـ r. وبينت نتائج التحليل الإحصـائي وجـود فـروق معنويـة بين المحطـات عدا المحطـة الثنانيـة والثالثة لم يسجل بينهما فرقاً معنوياً عند مستوى (P>0.05) وسجلت فروقاً معنوية بين جميع فصول السنة . جدول (1) A و B (معدلات التزاكيز العناصر النزرة (مايكروغرام/لتز) في

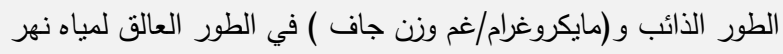
الفرات في منطقة الدراسة.

\section{(A)}

\begin{tabular}{|c|c|c|c|c|c|c|c|}
\hline \multicolumn{2}{|c|}{ الرصناصن Pb } & \multicolumn{2}{|c|}{ التُبكِل Ni } & \multicolumn{2}{|c|}{ الكأدميوم Cd } & \multirow{2}{*}{ الثحطة } & \multirow{2}{*}{ الموسم } \\
\hline الكالثئ & الألئب & الكالثي & الذائب & الكالثق & الألائب & & \\
\hline ir.a. & $\varepsilon . V$ & ह). & ND & 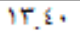 & Y. T. & 1 & \multirow{5}{*}{ خريف } \\
\hline$\sum Y, V$, & $\varepsilon$ & vo.lu & $\varepsilon . T$ & A.). & Q.T. & $Y$ & \\
\hline Vo.T. & $0 . \xi$. & $110 .$. & ND & YM.O. & T.a. & $T$ & \\
\hline$\varepsilon T, \lambda$. & $\varepsilon . \vee$ & $1+. v$. & ND & $11 .$. & r.a, & $\xi$ & \\
\hline 71.0. & Y... & 71.0. & Y1.0. & $\pi, v$ & $\therefore$. & 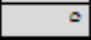 & \\
\hline 0.17 & 8.17 & $7 . \sqrt{2}$ & 0.17 & $1 \xi \sqrt{ } \varepsilon$ & $T .1 \xi$ & & المعتل \\
\hline$\xi 90\}$, & 7.1. & ir.a. & $\therefore \varepsilon$ & 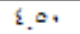 & ז.. & 1 & \multirow{5}{*}{ r.t } \\
\hline 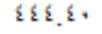 & 15.7 & V.. & 1.1 & $T . \cdot$ & .1 & $Y$ & \\
\hline ort.. & $A_{T}{ }^{\prime}$, & $1 . r 7$ & $1 . \varepsilon$. & T.Y. & • $\varepsilon$, & $r$ & \\
\hline qז., & ND & 19.5. & $T . \wedge$. & $r . \wedge$. & .9 , & $\xi$ & \\
\hline P.V. & 10.01 & FI.." & 1.14 & 1.1. & 9.14 & $\circ$ & \\
\hline To\{.0\} & $19.4 \mathrm{M}$ & Y7.10 & 1.07 & T.9Y & .8 , & & المعدل \\
\hline $11 \mathrm{Mr}$ & ND & $A \cdot \varepsilon$, & $T . Q$. & 11.0. & ND & 1 & \multirow{5}{*}{ رئِ } \\
\hline ire.". & 1.29 & ITt.V. & $1 . \mathrm{V}$ & $r h .$. & 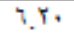 & $Y$ & \\
\hline $19 \lambda . T$. & $\mathrm{ND}$ & $18 \cdot a r$ & $\mathrm{ND}$ & Yo.s, & .. & $T$ & \\
\hline Tहl.. & $V_{.} \wedge \cdot$ & $104 .$. & V.1. & TV. $\varepsilon$, & V.1. & $\varepsilon$ & \\
\hline $1 \xi \lambda .$. & ND & $157 .$. & ND & $1 \pi .$. & T.Q. & $\therefore$ & \\
\hline 174.10 & $T .74$ & $1 \pi 4.01$ & E.r7 & Tr. & $\varepsilon \sqrt{2 \lambda}$ & & المعدل \\
\hline $1,4 .$. & IT.Q. & IVa.". & 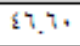 & TE., & $\mathrm{ND}$ & 1 & \multirow{5}{*}{ صنّف } \\
\hline ortr... & 19.00 & 9ov9... & ND & $\xi \xi .0$, & $\mathrm{ND}$ & $T$ & \\
\hline$\Delta \varepsilon \varepsilon, .$. & $r v . \varepsilon$ & 1991... & ND & $V \varepsilon, T$. & $\varepsilon \varepsilon$ & $T$ & \\
\hline 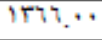 & rq.1. & $T T \varepsilon 1 .$. & $1 \varepsilon . T$ & TI.1. & T.Q. & $\varepsilon$ & \\
\hline Ta) V... & 10.7 & Trov... & $1, . \mathrm{vo}$ & $\xi \xi a$, & .9 . & $\therefore$ & \\
\hline Tri. & TE.Y & Eา7ห. & $1 E . T Y$ & $\varepsilon T . u$ & $1 . \sqrt{7}$ & & المعدل \\
\hline 9हV.IT & $1 \pi .10$ & ITYY.TV & $7 . T Y$ & $Y 1,1 T$ & 5.7. & & المعدل \\
\hline
\end{tabular}

$\mathrm{ND}=$ Not Detected

\section{Zنصر الخارصين Zn}

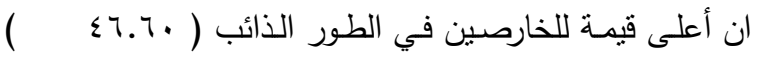

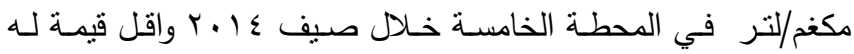

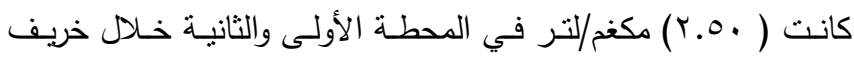

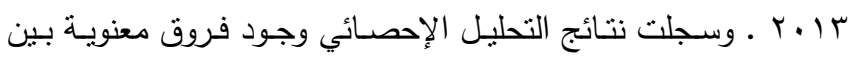

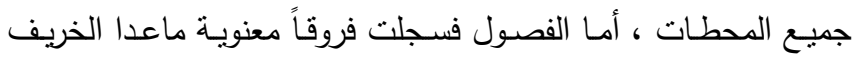
والثتاء لـم يظهر بينهمـا فـرق معنـوي عند مسـتوى (P>0.05). أمسا

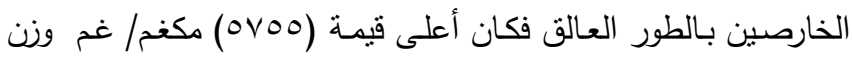

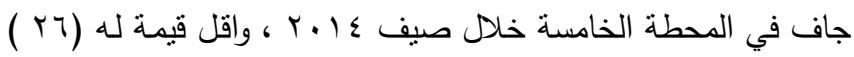

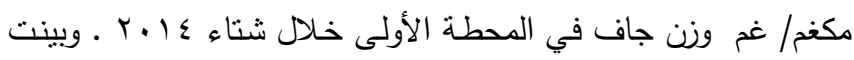
نتائج التحليل الإحصـائي وجود فروق معنويـة في قيم الخارصين بين المحطات ولجميع فصول السنة عند مستوى (P<0.05) .

عi Cu

تراوحت قيم النحساس مـن أعلى قيمـة في الطور الذائب (

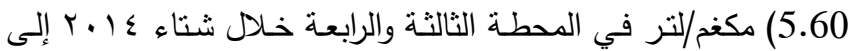
أدنى قيمة ظهرت في جميع المحطات خلال فصول السنة عدا الثتاء

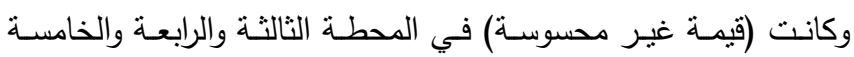

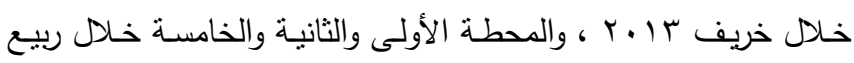

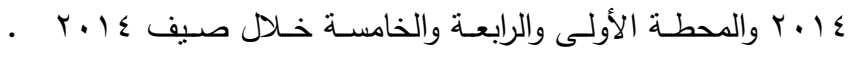
وأظهرت نتائج التحليل الإحصائي وجود فروق معنويـة بين المحطات ولات ولات

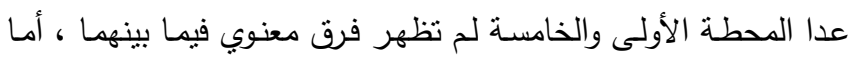

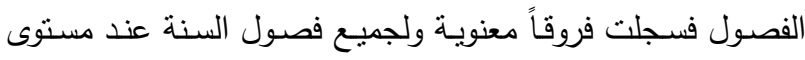

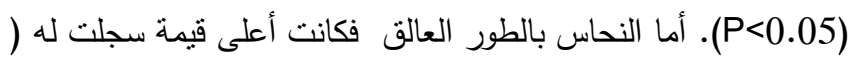

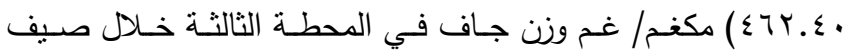

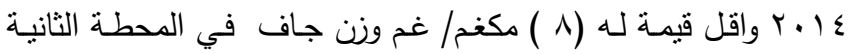

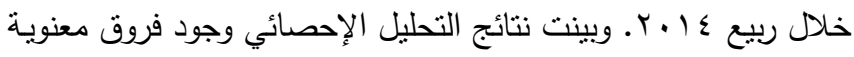

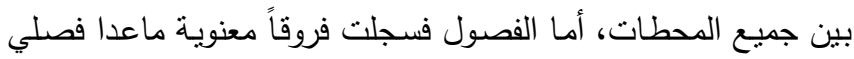
الخريف والربيع لم تسجل فرقا معنويا بينهما عند مسنتوى معنويـة .$(P<0.05)$

\section{Mn عنصر المنغتيز}

سـجلت أعلى قيمـة للمنغنيز في الطسور الـائب ( 44.10)

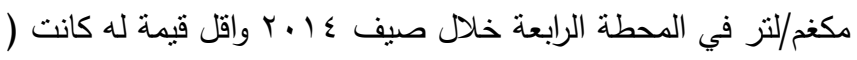

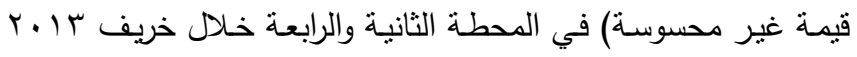

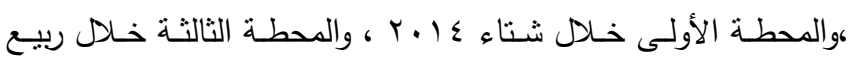


كما أوضحت نتائج الدراسة أن معدلات تراكيز العناصر النزرة بالطور

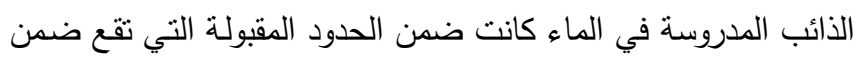

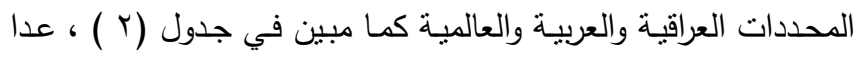

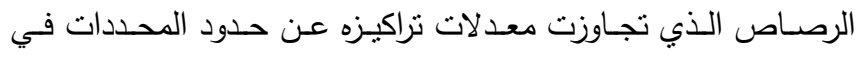
المواصفة العراقية لمياه الثرب ا ... وحدود المحددات الدولية لمياه

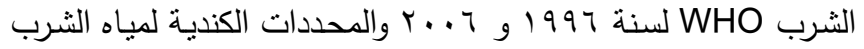
لسنة r . CDWQ الدراسات المحلية التي أجريت على نهر الفرات ونهر شط الحلة وشط

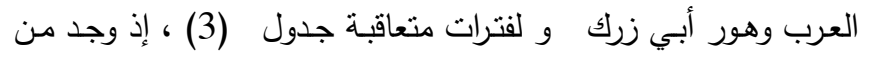

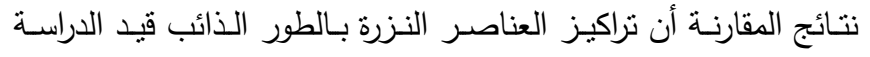

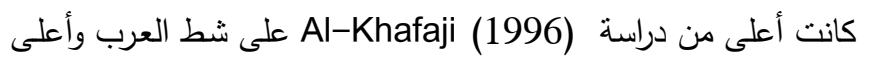

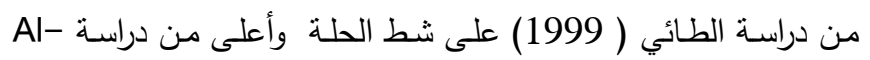

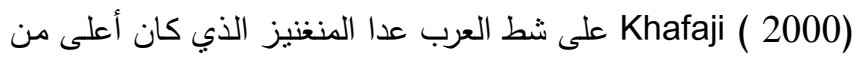

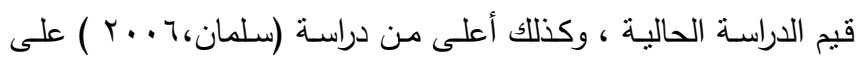

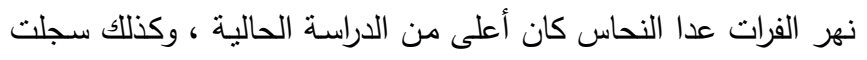

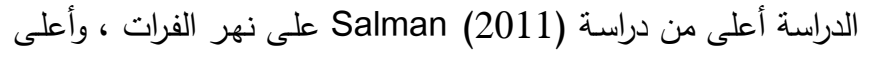

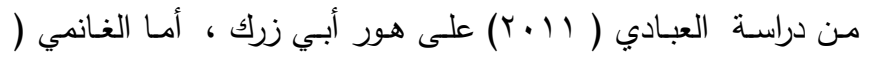

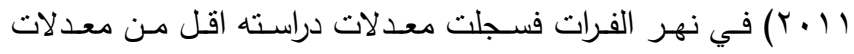

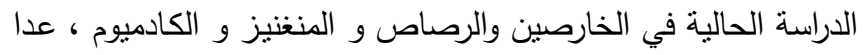

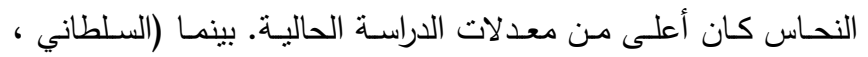

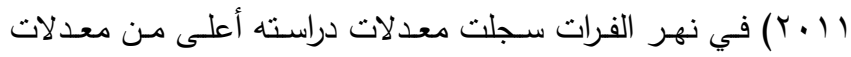

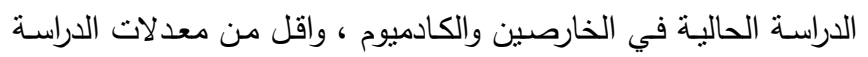

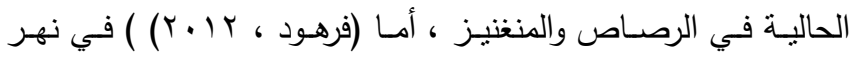

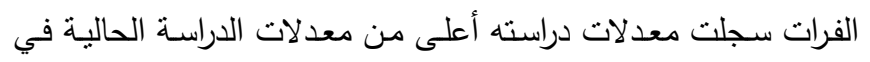

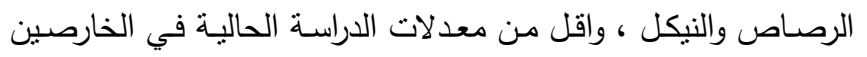

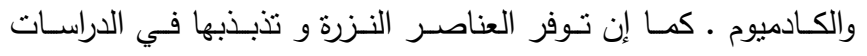

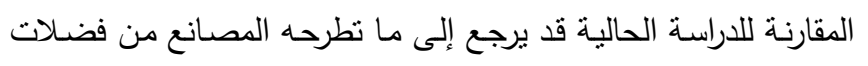

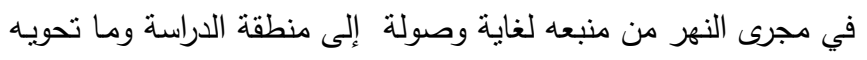

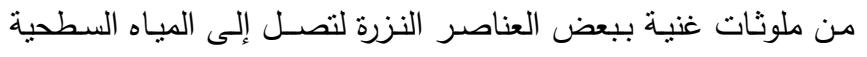

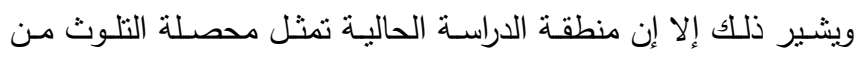

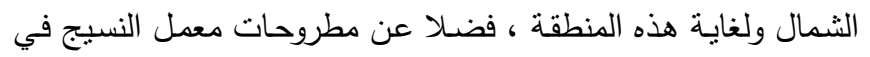

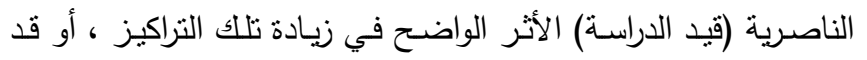

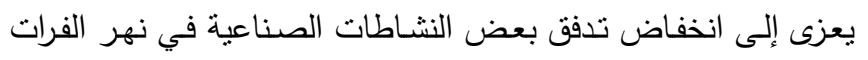
وإلى الزيادة في تصريف الفضـلات وخاصـة فضـلات المجاري المنزلية

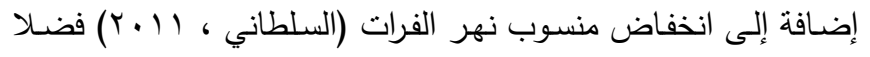

(B)

\begin{tabular}{|c|c|c|c|c|c|c|c|}
\hline \multicolumn{2}{|c|}{ ألمتتيز Mn } & \multicolumn{2}{|c|}{ التحاي Cu } & \multicolumn{2}{|c|}{ الخزعبن Zn } & \multirow{2}{*}{ |معقة } & \multirow{2}{*}{ المتوسيد } \\
\hline الملتى & ألقاتب & الملثق & القاتب & الملتى & أقانب & & \\
\hline m.1. & $6 .$. & EV.T. & r.t. & rav... & r.o. & 1 & \multirow{5}{*}{ זرا. } \\
\hline 7.1. & $\mathrm{ND}$ & TV.A. & 1.7. & st... & T.०. & $\%$ & \\
\hline 191.6. & $17 .$. & it.t. & $\mathrm{ND}$ & DA1... & $A .7$. & $r$ & \\
\hline$r \cdot \lambda_{.}$. & $\mathrm{ND}$ & ir.s. & $\mathrm{ND}$ & orn... & T. . . & 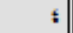 & \\
\hline $189 . .$. & 8.7. & $19 . .$. & $\mathrm{ND}$ & olv... & $y_{.} y_{.}$ & $\theta$ & \\
\hline ITo.Tr & 8.94 & 70.95 & .97 & 890.7. & 8.98 & & إلمغل \\
\hline of. . & $\mathrm{ND}$ & $16 .$. & $\because+1$ & rר... & O.r. & 1 & \multirow{5}{*}{ ثناء } \\
\hline M.t. & 16.8. & 16.0. & 1.6. & or... & O.A. & 7 & \\
\hline $181 . \pi$ & ET.T. & $11 . A$. & 0.7. & $n .$. & r. . & $F$ & \\
\hline \&1. ह. & Y.T. & IV.T. & 0.7. & จ9. द. & O.A. & 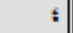 & \\
\hline$\pi .7$ & Ir.T. & 16.0. & T.7. & ir... & $6 .+1$ & 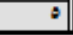 & \\
\hline oท. EA & 10.5. & $1 \varepsilon .8 \cdot$ & T.T\& & or.AA & $\varepsilon . M$ & & المغل \\
\hline$\Delta\{0 . T$. & $18 .$. & To.A. & $\mathrm{ND}$ & irra... & ir... & 1 & \multirow{5}{*}{ 1: } \\
\hline$\Delta 91, \ldots$ & $\varepsilon T . \ldots$ & $A \cdots$ & $\mathrm{ND}$ & $17.8 .$. & 17.8. & $\%$ & \\
\hline rr. & $\mathrm{ND}$ & M.A. & r.t. & var... & 11.4. & $r$ & \\
\hline rila... & $r \cdot . T$. & rr... & r.t. & ורי... & $A . \gamma$. & 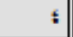 & \\
\hline זr... & rr... & 16.0. & $\mathrm{ND}$ & rva... & $1 . t$. & 8 & \\
\hline$r 9.91$ & 19.17 & Yo.ET & $1 . \pi r$ & $111 \pi .8$. & $11, y$. & & ألسئل \\
\hline $100, \ldots$ & $r \cdot A$. & of... & $\mathrm{ND}$ & $n+1, \ldots$ & (tr.). & 1 & \multirow{5}{*}{ ئيق } \\
\hline $111 .$. & $\mathrm{ND}$ & $1 \pi \mathrm{x} . \mathrm{r}$ & $0 . \ldots$ & olva... & TA. & $\%$ & \\
\hline $1.1 .$. & $\mathrm{ND}$ & str. & $0 . \ldots$ & हाור... & rr.A. & $r$ & \\
\hline$A .9, \ldots$ & \&.). & $r A, Y$. & $\mathrm{ND}$ & $1 \mathrm{~A} \cdot \mathrm{n}, \ldots$ & $19 .$. & $\leq$ & \\
\hline $9117 .$. & $\mathrm{ND}$ & rr.t. & $\mathrm{ND}$ & ovos... & 87.7 & $\theta$ & \\
\hline ๕9६.६. & $1 \pi .91$ & $1 \in T . E A$ & ז... & roti.s. & $\pi .91$ & & المغزل \\
\hline Ta.r. & $1 \pi . T$. & or.m & $1, \mathrm{AA}$ & IraA.Tr & $1 T . A Y$ & & الصغئ \\
\hline
\end{tabular}

$\mathrm{ND}=$ Not Detected

\section{المناقشة :}

تتواجد العناصر النزرة في المياه إمـا بطور ذائب أو مرتبط مـع

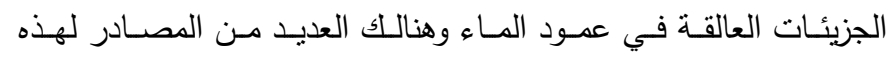
العناصر في البيئة المائية منها ما هو طبيعي مثل التعرية والتجوية للتربة

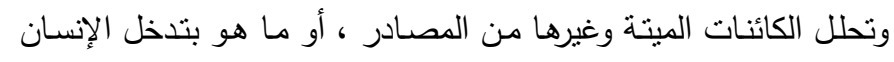

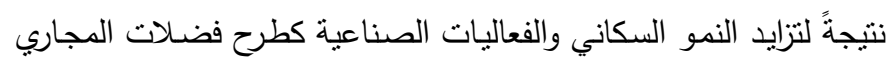

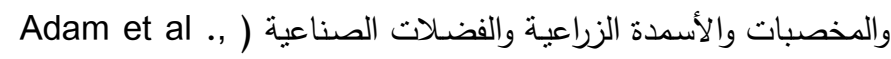

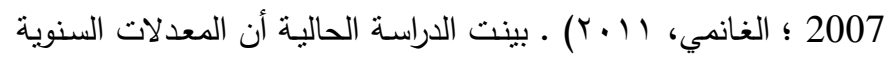

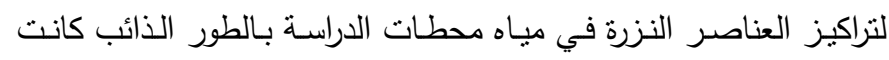

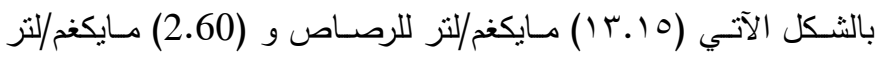
للكادميوم و(13.87) مايكغم/لتز للخارصين و (6.32) مايكغم/لتز للنيكل

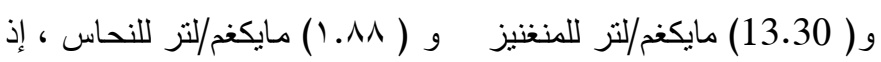
كان ترتيب نركيز هذه العناصر الذائبة بالثكل الآتي : . $\mathrm{Cu}<\mathrm{Cd}<\mathrm{Ni}<\mathrm{Pb}<\mathrm{Mn}<\mathrm{Zn}$ 
الإضـاءة (2011) Salman أمـا سـبب انخفاض تراكيز بعض

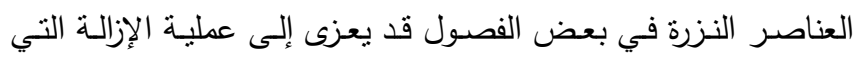

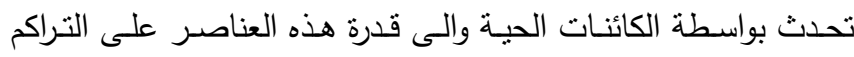
داخل أجسام الكائنات الحية أو إلى أدمصاصها بواسطة المواد العالقة وبالتالي ترسيبها ( الحيالي ، 2001) ـ كما إنَّ تركيز العناصر النزرة يـزداد فـي المواسـم الجافــة والحسارة بسـبب ارتفــاع درجـة الحــرارة ( Y.11، Abd \&Musa 2009 ؛ Obasohan , 2008

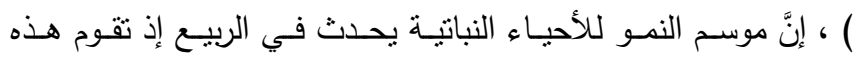

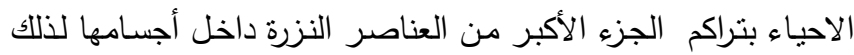

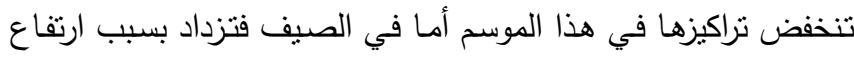
درجات الحرارة مما يؤدي إلى زيادة في معدلات التبخر وتحلل المواد

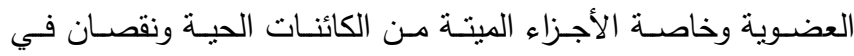

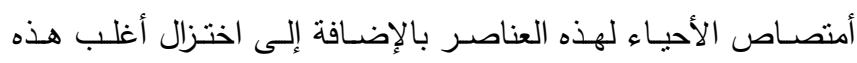

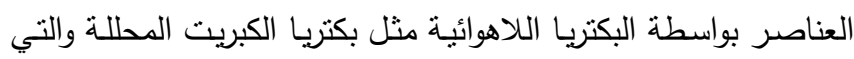

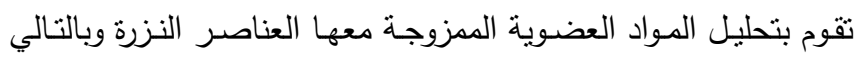
سوف تؤدي إلى أحداث زيادة في تركيز العناصر النزرة الذائبة في الماء

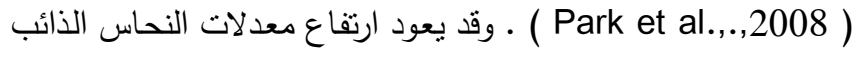

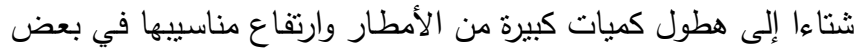
المناطق السكنية مما أدى إلى تصـريفها عبر آليات تابعـة للمديريات

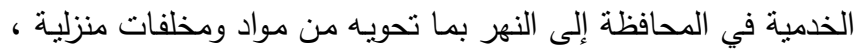

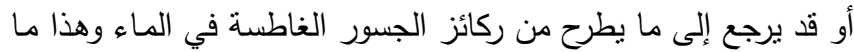

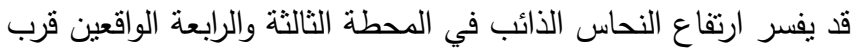
جسر السريع الكونكريني وجسر الزينون على التوالي خلال فصل الثتاء

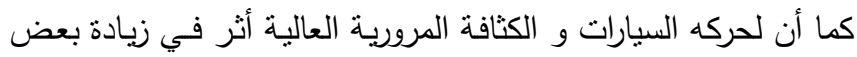

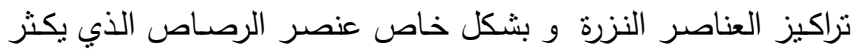
في عوادم السيارات (Rajendran et. al., 2005 ) ، وهذا ما أكدنه دراسـة (2005) Al-Khafaji على نهر الفرات عند مدينة الناصرية . أو قد يعزى إلى محطة غسيل السيارات الواقعة قرب المحطة الثالثة وما تطرحسه إلى النهر من مخلفات السيارات بعد الغسيل وهذا مـا يفسر تسجيل معدلات عاليه للرصاص العالق والنحاس العالق في المحطة

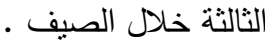

عن ذلك عدد ساعات العمل أو توقف المعمل ذاته في بعض الأحيان

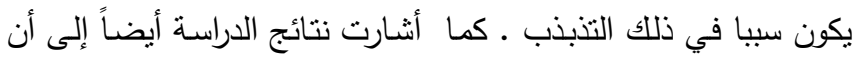
معدلات تراكيز العناصر النزرة بالطور العالق في مباه نهر الفرات كانت

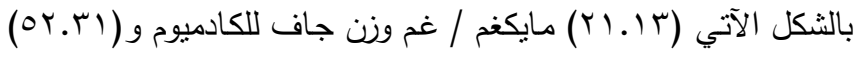

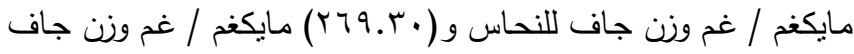

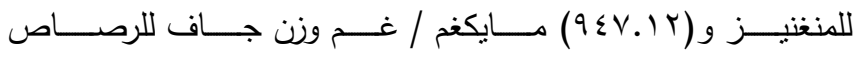

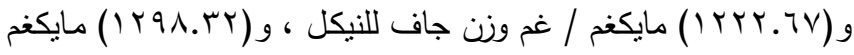

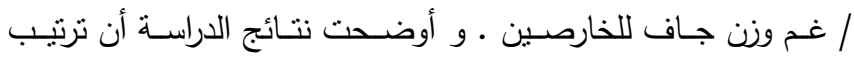
تراكيز العناصر النزرة في الطور العالق للمياه كانت بالثكل الآتي :الخارصين > النيكل > الرصاص > المنغنيز > النحاس > الكادميوم .

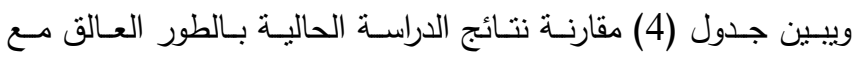

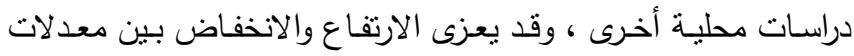

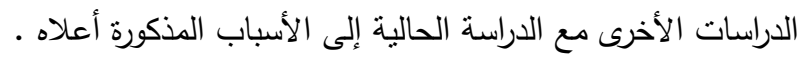

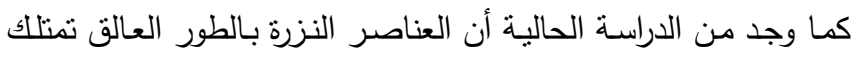
تركيز أعلى من الطور الذائب وقد يرجع ذلك إلى توزيع العناصر النزرة

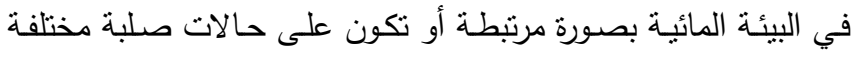
لذلك فأن فترة بقائها بشكل ذائب تكون قصيرة جداً مما يقلل نراكيزهـا لذلك نجد تفوق الطور العالق على الطور الذائب في هذه الدراسة وهذا يتفق مع ما ذكره (جبر،r. . . . وقد يعزى السبب في ذلك إلى الميل العالي لهذه العناصر إلى الادمصـاص والارتباط على سطوح المواد العالقة والمواد العضوية والطين في عمود الماء ل Demina ,et al.,

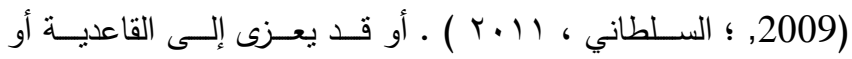

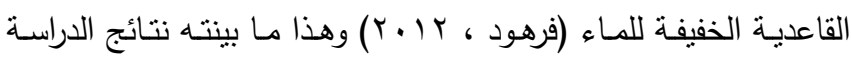

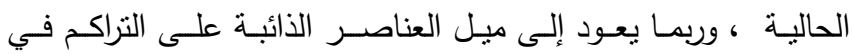

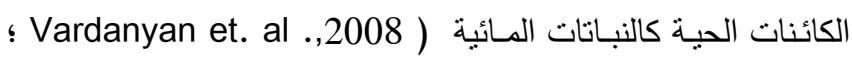

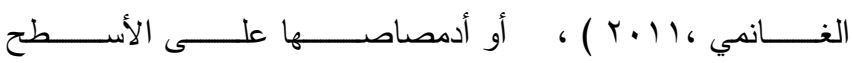

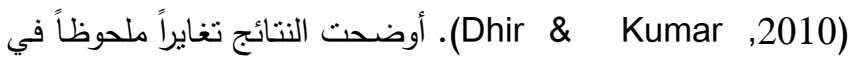

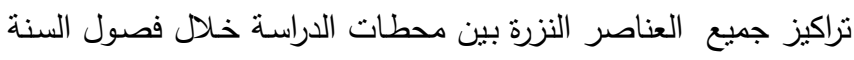

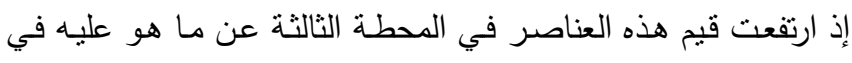
المحطات الأولى والثانية والرابعة والخامسة ، وقد يعزى ذلك إلى التتوع في مصادر التلوث بهذه العناصر أو إلى اختلاف كمية المواد الملوثة المتصرفة إلى النهر وارتفاع درجة الحرارة مما يؤدي إلى التبخر (الطائي

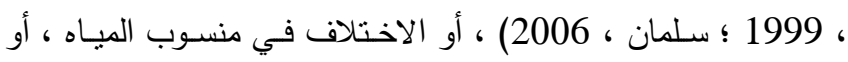

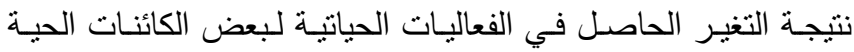
والتي تتأثر بواسطة عدة عوامل منها كمية الغذاء والتكاثر وطول مدة 
يستتنج مما تقدم والنتائج التي تم الحصول عليها خلال الدراسة الحالية إن

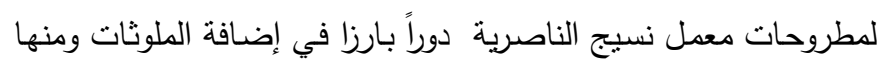

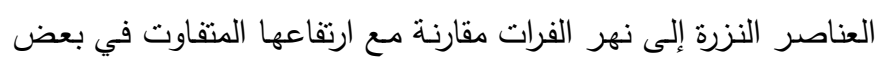

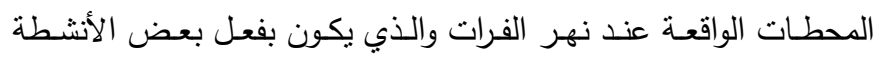

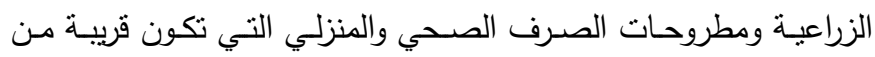
محطات الدراسة ، كما سجلت نركيز العناصر النزرة في الطور العالق أعلى مما هو عليه في الطور الذائب.

\section{References}

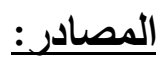

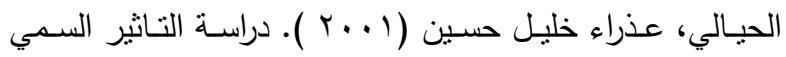

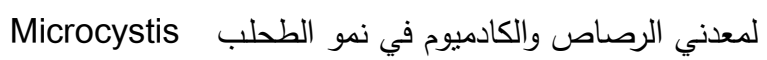

aeruginosa kuetz

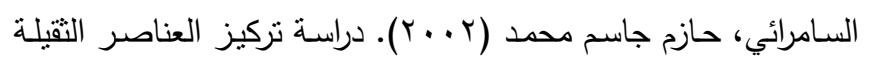

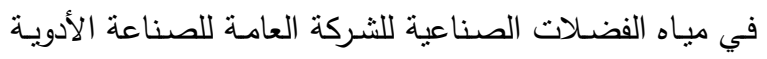
والمستلزمات الطبية في سامراء وإيجاد المعالجات اللازمة لها ،رسالة ماجستير، كلية الهندسة جامعة تكريت.

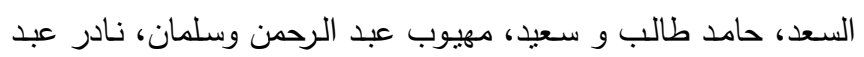

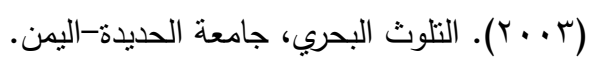

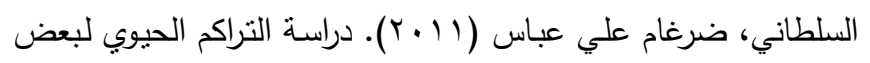

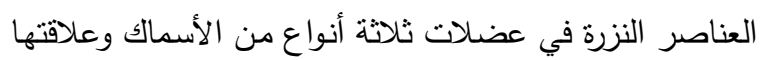
بتغاير العوامل البيئية في نهر الفرات / وسط العراق. رسـالة ماجستير، كلية العلوم، جامعة بابل.

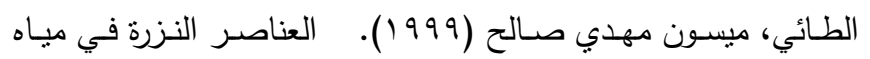
ورواسب واسماك ونباتات نهر شط الحلة. اطروحه دكتوراه، كلية العلوم، جامعة بابل.

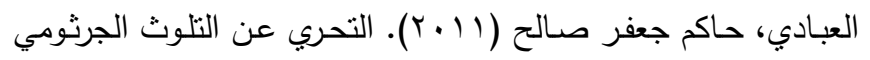
والمعدني في مياه ورواسب هور ابي زرك جنوب العراق. رسالة

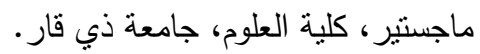

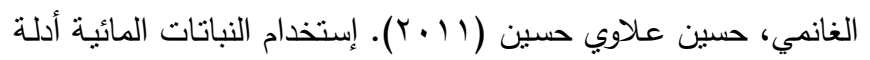
حياتية على التلوث بالعناصر التقيلة في نهر الفرات - العراق. رسالة ماجستير، كلية العلوم، جامعة بابل.

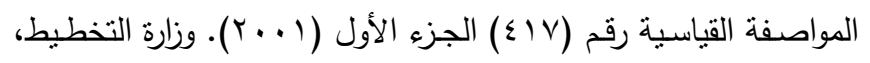
الجهاز المركزي للتقييس والسيطرة النوعية، الجمهورية العراقية.
جدول (r) : مقارنة بين تراكيز العناصر النزرة الذائبة الدروسة (مايكرو غرام / لتر ) في مياه محطات الدراسة مع بعض المناصن المحددات العراقية

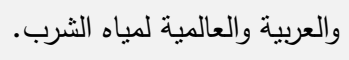

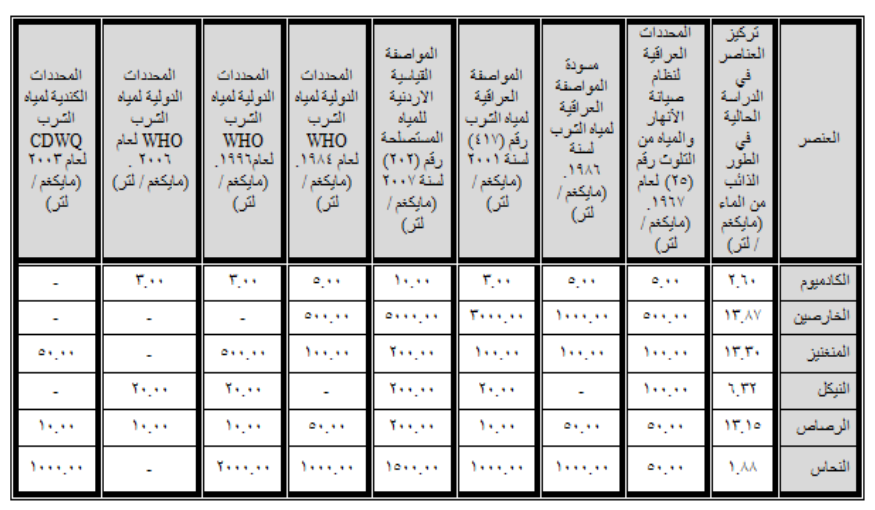

جدول (ץ) مقارنة بين معدلات تراكيز العناصر النزرة في الماء بالطور

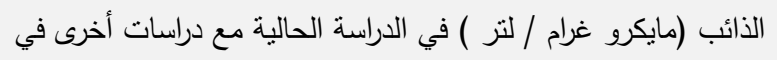
مناطق مختلفة من العراق

\begin{tabular}{|c|c|c|c|c|c|c|c|}
\hline النصائر & الخارعبن & الثرصناص & النيكل & المنثيز & النحسا & الكاثمبوم & \\
\hline Al-Khafaji, , 199Y) & 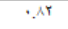 & $\because T$ & $r . \Lambda T$ & 1.04 & $\cdot .8 \mathrm{~V}$ & .19 & تط العرب ـ العرائ \\
\hline (الطاتلى، (1949) & A.VT & 8,1 & $\cdot . \mathrm{YV}$ & .97 & 1.11 & $\therefore 11$ & نهير العلك العرافي \\
\hline Al-Khafaji ,,$\cdots$ & $\varepsilon \%$ & $\because n$ & - & 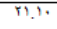 & 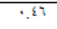 & $\because \mu$ & تُط العرب ـ العرائي \\
\hline 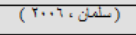 & $1 .$. & $\because$ & $\because \cdot \mathrm{v}$ & $4 \pi$ & $r . \varepsilon$ & 5 & نهير النراكــالترأق \\
\hline$(9 \cdots 1,4)$ & 18,10 & - & ror & - & $1 ., y$ & ri. & لنهر الغرانـ ـ العرائي \\
\hline 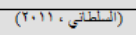 & 19.74 & 1.419 & 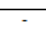 & 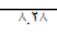 & $\cdot$ & T.YST & انهر التراثـــالعرأي \\
\hline (العبادي ، 1 (1+r) & 0.81 & 1.7 & 1.10 & - &.$\circ r$ & $\because r$ & هور أبي زرك- -جنوب الترائي \\
\hline 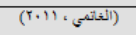 & - & $\because r 1$ &. $\mathrm{r}$ & $\varepsilon, Y 9$ & 7.87 & - & 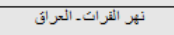 \\
\hline (Salman, ,'+'!) & $19 . y_{0}$ & $. .1 \leqslant 4$ & $\because \%$ & $r .99$ & - & $\because$ & انهر الثراكـــالعرائ \\
\hline 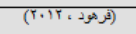 & $\because . r$ & $\pi$ & V.r & - & - & T.YY & نهير الثراكــاكترائ \\
\hline الدرسة أدالية & IT,AV & 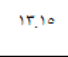 & $8 \pi r$ & ir.T. & i.从 & rat & ركز مدينة الثناهرية \\
\hline
\end{tabular}

جدول ( ؛ ) مقارنة بين معدلات تراكيز العناصر النزرة في الماء بالطور

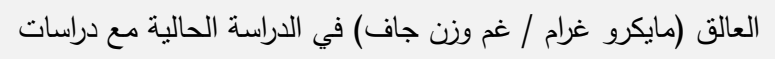
أخرى في مناطق مختلفة من العراق.

\begin{tabular}{|c|c|c|c|c|c|c|c|}
\hline المصادر & الخازمسن & 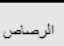 & النزيكل & المنتفزي & النحفا & الكانميوم & العنصر \\
\hline (Al-Khafaji, , 1947) & TEहa & 8.9 & $1,1,1 / 2$ & To.00 & TIV..0 & ह7.Tा & 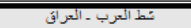 \\
\hline (الطأئى، ، 1999) & r.o..0 & or.1h & r), $1 \varepsilon$ & $\sqrt{n, E S T}$ & $0 ! 11$ & $r .09$ & نهر الحلة ـ العرأي \\
\hline$($ Al-Khafaji,$r \cdots)$ & Tr19.0Y & TY,AT & - & Tor.lY & \&1.0\% & $\lambda \mu$ & تُط العرب ـ ألعرائ \\
\hline 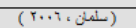 & 09,91 & $\because \therefore$ & .19 & $\pi \mathrm{rV}$ & 15,90 & $1, Y Y$ & نهير الثراتــــأعرأق \\
\hline (Salman, Y.'I) & $\because \because, 1$ & $\because .04$ & .199 & TQ.00 & - & $1 \cdot 19$ & نهر الثراتــ العرأ \\
\hline (الكلطانتي، ، (1, (r) & $1 \xi 1, \xi \mathrm{V}$ & $r \wedge .91$ & - & $10.1 \mathrm{Y}$ & - & 78.79 & نهر الثراتـــ العرأي \\
\hline (العبادي ، '11) & $\pi .54$ & $r$ II, N & $\because .11$ & - & v.M & O.YY & هور أبي زرئـــبنوب الثراف \\
\hline 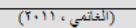 & - & $\because 4$ & 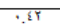 & $0 . \cdot 4$ & V.TY & - & نهر الثراثـــالعرأق \\
\hline 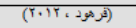 & Tre.T & $17+1.87$ & IET.\&T & - & - & $17.7 \mathrm{~V}$ & نهر التراتيـ العرأي \\
\hline الثرسة الدالية & Ir9人.Tr & १\&V.,Y & אידוזיו & זד.T. & or, $T$ r & $T, 1 T$ & نهركز الثرات - العرائة \\
\hline
\end{tabular}


Hillawi Cultivars of date palms. Marsh Bulletin, 4 (1): 85-97.

Adam, R.S. ; Al-Shawi, I.J.M. and Al-Imarah, F.J.M. (2007). Distribution of some chemical elements in the marsh lands of southern Iraq after rehabilitation . Mash Bulletin, 2 (1): 1117.

Al-Khafaji, B.Y. (1996). Trace metals in water, sediments, and fishes from Shatt Al- Arab estuary north-west Arabian Gulf. Ph.D. Thesis, College of Education, Basra University.

Al-Khafaji, B.Y. (2000). Preliminary survey of selected heavy metals in AL-Jubayla creek connected with Shatt AL-Arab river. Marina Mesopotamica, 15 (1): 69-80.

Al-Khafaji, B.Y. (2005). Trace elements distribution in the Euphrates river near Al-Nassiriya city southern part of Iraq. J. of Karbala university (accepted to publisher).

Al-Khafaji, B.Y. (2010). Distribution of Some Heavy Metals In The Euphrates River Ecosystem Near Al-Nassiriya City Center South Iraq. J. Thi-Qar Sci., 2 (2): 11-24.

American Public Helth Association (APHA) (2003). Standard methods for examination of water and wastewater.(20th Ed.). Washington DC,USA.

Canadian Drinking Water Quality (CDWQ) (2003). Guidelines for Canadian drinking water quality. Federal-Provicial-Territorial Committee on Environmental and Occupational Health, PP: 110.

Demina, L.L. ; Galkin, S.V. and Shumilin, E.N. (2009). Bioaccumulation of some trace elements in the biota of hydrothermal fields of the Guaymas Basin (Gulf of California). Boletin De LA Sociaeded Geologica Mexicana, 61 (1): 31-45.

Dhir, B. and Kumar, R. (2010). Adsorption of heavy metals by Salvinia biomass and agricultural residues. Int. J. Environ. Res., 4 (3): 427-432.

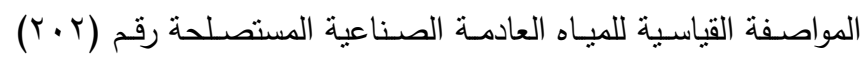

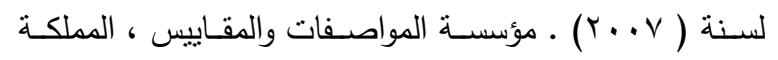

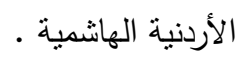

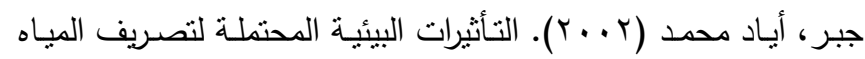
الصناعية على الهائمات النباتية، رسالة ماجستير، كلية العلوم،

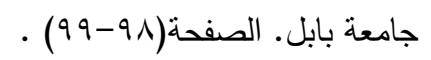

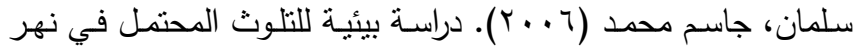

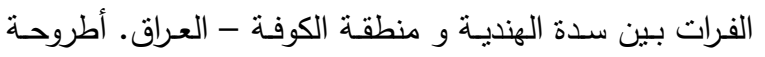
دكتوراة، كلية العلوم، جامعة بابل.

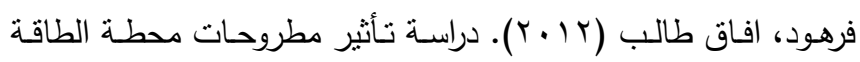

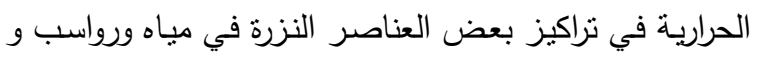

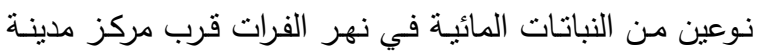

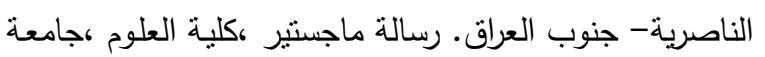

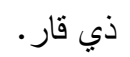
فهد، كامل كاظم (T . T). مستح بيئي لمياه الجزء الجنوبي من نهر

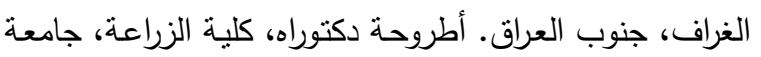

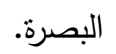

موسى، سهير ازهر و علي، اسماء عبد (910 (1). تلكوث نهر دجلة

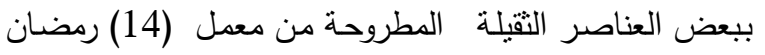
للغزل والنسيج في الكاظمية. مجلة بحوث علوم الحياة، 17 rr-וT:

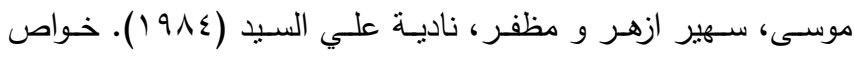
الفضلات السائلة المطروحة من معدل الغزل والنسيج الصوفي نادئه

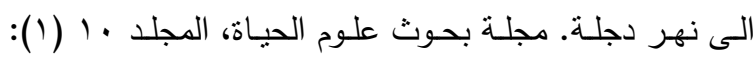
$.47-17$

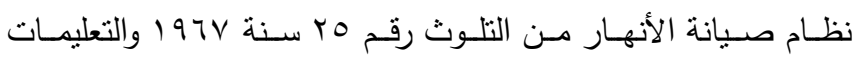

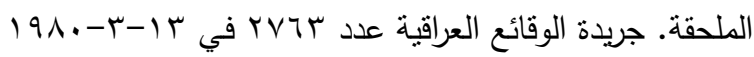

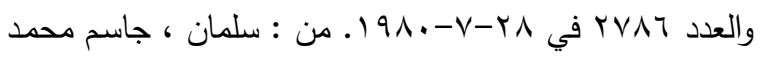

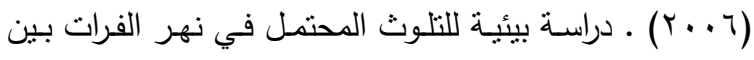
سـدة الهنديـة ومنطقـة الكوفـة-العـراق. أطروحـة دكتوراه، كليـة

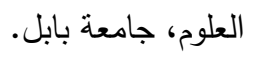

Abd, A.K.M. and Musa, Z.J. (2009). A study of levels of trace elements in water and soil of Hamadan Canal and Its Effect on leaves fruits of Braim and 
Environmental Research and Assessment, Bucharest, Romania, March, 23-27.

Vardanyan, L. ; Schider, K. ; Sayadyan, H. ; Heege, T. ; Heblinski, J. ; Agyemang, T. and De, J.J. (2008). Heavy metal accumulation by certain aquatic macrophytes from lake Seven (Armenia ). In: Sengupta, M. and Dalwani, R. (Eds.), The 12 th World Lake Conference, PP: 1028-1038.

World Health Organization (WHO) (1984). Guideline for Drinking Water Qality , WHO, Swiss, Geneva.

World Health Organization (WHO) (1996). Guideline for Drinking Water Qality. (2nd ed.). Health Criteria and other Supporting Information, Geneva.

World Health Organization (WHO) (2006). Guidelines for drinking- water quality. (3rd ed.). First addendum, Geneva, 1: 515.
Maryland Department of Environment (MDE) (2003). Water Qality Analysis of heavy metals for the Loch Raven Reservoir impoundment in Baltimore county, Maryland. US Environmental Protection Agency, Cincinnati, Ohio.

Obasohan, E.E. (2008). The use of heavy metal load as an indicator of the suitability of the water and fish of Ibiekuma Stream for domestic and consumption purposes. African J. of Biotechnology, 7 (23): 4345-4348.

Park, N. ; Kim, J.H. and Cho, J. (2008). Organic matter, anion and metal wastewater treatment in Damyang surface flow constructed wetlands in Korea. Ecological Engineering Journal, 32 (1): 68-71.

Percy, J.A. (2004). Contaminant Concerns: Heavy Metals and the Bay of Fundy. Fundy Issues \#25. Bay of Fundy Ecosystem Partnership Publication, P12.

Rajendran, R.B. ; Imagawa, T. ; Tao, H. and Ramesh, R. (2005). Distribution of PCBs, HCHs and DDTs and their ecotoxicological implications in Bay of Bengal, India. Envi. In., 31 (4): 503-512.

Riley, J.P. and Taylor, D.T. (1968). Chelating resins for the concentration of trace elements from sea water and their analytical use in conjuction with atomic absorption specrtophotometry. Anal. Chim. Acta., 40: 479-485.

Salman, J.M. (2011). The Clam Pseudodontpsis euphraticus (Bourguignat, 1852) as a bioaccumulation indicator organism of heavy metals Euphrates River, Iraq. J. Babylon Uni./ Pure and Applied Sciences, 19 (3): 884-893.

Sturgeon, R.E. ; Desaulincrs, J.A. ; Berman, S.S. and Russell, D.S. (1982). Determination of trace metals in estuarine sediment by graphite fernace atomic absorption spectrophotometry. Anal. Chem. Acta., 134: 288-291.

Tokalioglu, S. ; Kartal, S. and Elci, L. (2000). Speciation and determination of heavy metals in lake waters by atomic absorption spectrometry after sorption on amberlite XAD-16 Resin. Analytical Sciences, 16: 1169-1174.

Ulmanu, M. ; Anger, I. ; Lakatos, J. and Aura, G. (2003). Contribution to some heavy metals removal aqueous solution using peat. Proceeding of the First International Conference on 